\title{
Two New Factors of Fermat Numbers
}

\author{
By John C. Hallyburton, Jr. and John Brillhart \\ Dedicated to D. H. Lehmer on his 70 th birthday
}

\begin{abstract}
A new prime factor is given for each of the Fermat numbers $F_{12}$ and $F_{13}$ (none was previously known for $F_{13}$ ). The factoring method used and its machine implementation are discussed. A short table of factors and a current status list are also included.
\end{abstract}

In recent years various investigators have used computers to search for prime factors of the Fermat numbers $F_{m}=2^{2^{m}}+1, m \geqslant 7$ (see Selfridge [10], Robinson [7]-[9], Riesel [6], Brillhart [1], Wrathall [12], Morrison and Brillhart [3] , [4] ). In our investigation we have found two new factors, namely:

$190274191361=11613415 \cdot 2^{14}+1$ and $2710954639361=41365885 \cdot 2^{16}+1$, which divide $F_{12}$ and $F_{13}$, respectively. Previously, three prime factors of $F_{12}$ had been discovered, while $F_{13}$ was only known to be composite (see Paxson [5]).

It is well known that any prime factor of $F_{m}$ has the form $k \cdot 2^{m+2}+1$, $m \geqslant 2$. In searching for such a factor, we can try dividing $F_{m}$ by each $d_{k}$ of this form for $k$ less than some search limit $L_{m}$. Many composite $d_{k}$ can, of course, be eliminated as trial divisors in advance by sieving on the arithmetic sequence $\left\{d_{k}\right\}$ with small, odd primes (in our program the odd primes less than 500 were used).

To discover whether a $d_{k}$ which has survived the sieving is a factor of $F_{m}$, we calculate $F_{m}\left(\bmod d_{k}\right)$ by the usual powering method-here only a sequence of squarings and reductions.

Since the residues $r_{i}$ of the powers $2^{2^{i}}\left(\bmod d_{k}\right), i \leqslant m$, will necessarily be computed in this calculation, it is clear we should check to see if $r_{i} \equiv-1\left(\bmod d_{k}\right)$ for each $i, 8 \leqslant i \leqslant m$, thereby determining in one stroke if $d_{k}$ is a factor of any of these $F_{i}$. It should be remarked, however, that $k$ is always taken to be odd. Thus, if $F_{m}$ has a prime factor $k \cdot 2^{n+2}+1$ with $k$ odd and $n>m$, then this factor can only be discovered during the search for factors of $F_{n}$. For example, the factor of $F_{13}$ was found during the investigation of $F_{14}$, since in this case $n-m$ $=1$.

This procedure was coded in COMPASS assembly language for the CDC 6400 at

Received May 20, 1974.

AMS (MOS) subject classifications (1970). Primary 10A25, 10A40; Secondary 10-04.

Key words and phrases. Fermat numbers, factoring. 
TABLE 1. Search limits

\begin{tabular}{cl}
\multicolumn{2}{c}{$1 \leqslant k \leqslant L_{m}, \quad k$ odd } \\
\hline$m$ & \multicolumn{1}{c}{$L_{m}$} \\
\hline 8 & 1542455295 \\
$9-13$ & 16777215 \\
14 & 792008373 \\
$15-22$ & 16777215 \\
\hline
\end{tabular}

TABLE 2. Factors of $F_{m}, 5 \leqslant m \leqslant 22$

\begin{tabular}{|c|c|c|c|}
\hline$m$ & Prime Factors & Date & Discoverer \\
\hline 5 & 641 & 1732 & Euler \\
\hline 5 & 6700417 & 1732 & Euler \\
\hline 6 & 274177 & 1880 & Landry \\
\hline 6 & 67280421310721 & 1880 & Landry, LeLasseur, Gérardin \\
\hline 7 & 59649589127417217 & 1970 & Morrison, Brillhart \\
\hline 7 & 5704689200685129054721 & 1970 & Morrison, Brillhart \\
\hline 8 & c & 1909 & Morehead, Western \\
\hline 9 & 2424833 & 1903 & Western \\
\hline 9 & $\mathrm{c}^{*}$ & 1967 & Brillhart \\
\hline 10 & 45592577 & 1953 & Selfridge \\
\hline 10 & 6487031809 & 1962 & Brillhart \\
\hline 10 & $c^{*}$ & 1967 & Brillhart \\
\hline 11 & 319489 & 1899 & Cunningham \\
\hline 11 & 974849 & 1899 & Cunningham \\
\hline 12 & 114689 & 1877 & Lucas, Pervouchine \\
\hline 12 & 26017793 & 1903 & Western \\
\hline 12 & 63766529 & 1903 & Western \\
\hline 12 & 190274191361 & 1974 & Hallyburton, Brillhart \\
\hline 13 & 2710954639361 & 1974 & Hallyburton, Brillhart \\
\hline 14 & c & 1961 & Selfridge, Hurwitz \\
\hline 15 & 1214251009 & 1925 & Kraitchik \\
\hline 16 & 825753601 & 1953 & Selfridge \\
\hline 17 & $?$ & & \\
\hline 18 & 13631489 & 1903 & Western \\
\hline 19 & 70525124609 & 1962 & Riesel \\
\hline 19 & 646730219521 & 1963 & Wrathall \\
\hline 20 & $?$ & & \\
\hline 21 & 4485296422913 & 1963 & Wrathall \\
\hline 22 & $?$ & & \\
\hline
\end{tabular}

$?=$ character of $F_{m}$ is unknown.

$c=$ number is composite. ( $*=$ previously unpublished results found on the IBM 7094 at the Bell Telephone Laboratories at Holmdel, New Jersey.) 


\begin{tabular}{ll}
\hline \multicolumn{1}{c}{$m$} & \multicolumn{1}{c}{ Character of $F_{m}$} \\
\hline $0,1,2,3,4$ & Prime \\
$5,6,7$ & Composite and completely factored \\
$10^{+}, 11,12^{*}, 19,30,38$ & $\begin{array}{l}\text { Two or four factors known } \\
(+=\text { cofactor is composite })\end{array}$ \\
$9^{+}, 13,15,16,18,21,23,25,26,27$, & Only one prime factor known \\
$32,36,39,42,52,55,58,63,73,77$, & \\
$81,117,125,144,150,207,226,228$, & \\
$250,267,268,284,316,452,1945$ & \\
8,14 & Composite but no factor known \\
$17,20,22,24,28,29,31$, etc. & Character unknown \\
\hline
\end{tabular}

the University of Arizona Computer Center and was run for 150 hours at lowest priority.

It should be pointed out that there is a problem with running a low-priority job, which requires considerable memory, on a large processor like the CDC 6400. To maximize running time the program should always remain in core, i.e., it should not use so much space that other jobs (all of higher priority) continually cause it to be swapped onto a disk because of their memory requirements. On the other hand, the sieve, which the program uses, should occupy enough memory to avoid its continually having to be remade.

To solve this problem, we usually ran the program only on weekends, holidays, or late at night (when only nightowl system programmers were around). By asking, we could get a good idea of their memory requirements and scale our own space request accordingly. In addition to this, pieces of the sieve (64 words at a time-the minimum possible on this machine) were returned to the system, after they had been read, to further increase the likelihood that the job would remain in core. (The sieve was stored in reverse order, adjacent to the program, to facilitate this return.) When reading the sieve was completed, a new memory request was automatically made and a new section of the sieve was constructed. In general, the sieve required between half a million and a million bits.

In this investigation only $F_{m}$ for $8 \leqslant m \leqslant 22$ were considered. The search limits on $k$ that were used in each case are listed on Table 1. A limitation of $2^{48}$ was placed on $d_{k}=k \cdot 2^{m+2}+1$ so that a remainder ( $\left.\bmod d_{k}\right)$ would be less than this amount-a convenient size for double-precision squaring on the CDC 6400 .

All known factors of these $F_{m}$ were rediscovered and are given with their discoverer and date in Table 2 (dates after 1925 are the dates of discovery).

Acknowledgements. The authors would like to express their gratitude to the staff and management of the University of Arizona Computer Center for their support of this project. 
Department of Computer Science

University of Arizona

Tucson, Arizona 85721

Department of Mathematics

University of Arizona

Tucson, Arizona 85721

1. JOHN BRILLHART, "Some miscellaneous factorizations," Math. Comp., v. 17, 1963, pp. $447-450$.

2. ALEXANDER HURWITZ \& J. L. SELFRIDGE, “Fermat numbers and perfect numbers," Notices Amer. Math. Soc., v. 8, 1961, p. 601, Abstract \#587-104.

3, MICHAEL A. MORRISON \& JOHN BRILLHART, "The factorization of $F_{7}$," Bull. Amer. Math. Soc., v. 77, 1971, p. 264. MR $42 \# 3012$.

4. MICHAEL A. MORRISON \& JOHN BRILLHART, "A method of factoring and the factorization of $F_{7}$," Math. Comp., v. 29, 1975, pp. 183-205 (this issue).

5. G. A. PAXSON, "The compositeness of the thirteenth Fermat number," Math. Comp., v. 15, 1961, p. 420. MR 23 \#A 1578.

6. HANS RIESEL, "A factor of the Fermat number $F_{19}$," Math. Comp., v. 17, 1963, p. 458.

7. RAPHAEL M. ROBINSON, "Mersenne and Fermat numbers," Proc. Amer. Math. Soc., v. 5, 1954, pp. 842-846. MR 16, 335 .

8. RAPHAEL M. ROBINSON, "Factors of Fermat numbers," $M T A C$, v. 11, 1957, pp. 21 22. MR 19, 14.

9. RAPHAEL M. ROBINSON, "A report on primes of the form $k \cdot 2^{n}+1$ and on factors of Fermat numbers," Proc. Amer. Math. Soc., v. 9, 1958, pp. 673-681. MR 20 \#3097.

10. J. L. SELFRIDGE, "Factors of Fermat numbers," $M T A C$, v. 7, 1953, pp. 274-275.

11. J. L. SELFRIDGE \& ALEXANDER HURWITZ, "Fermat numbers and Mersenne numbers," Math. Comp., v. 18, 1964, pp. 146-148. MR 28 \#2991.

12. CLAUDE P. WRATHALL, "New factors of Fermat numbers," Math. Comp., v. 18, 1964, pp. 324-325. MR $29 \# 1167$. 